J. clin. Path. (1964), 17, 338

\title{
Thrombolytic therapy
}

\author{
GEORGE P. MCNICOL AND A. S. DOUGLAS
}

From the University Department of Medicine, Royal Infirmary, Glasgow

The purpose of this communication is to review briefly the agents available at the present time for use in thrombolytic therapy and to indicate why we believe that at the present time streptokinase is the only suitable agent for extended clinical trial. An account will then be given of the use of streptokinase administered by local perfusion in the treatment of peripheral arterial occlusion and finally some of the problems in the field of thrombolytic therapy will be briefly discussed.

\section{ACTIVATOR OR PLASMIN?}

Turning first to the agents available, one of the controversial areas in the field of fibrinolytic therapy is whether it is more rational to use activators or preformed plasmin as a therapeutic agent. The evidence has been reviewed by Sherry and Fletcher (1960) and by Sawyer, Alkjaersig, Fletcher, and Sherry (1961), and the case for the use of activator seems well established. It would seem likely that the mechanism of fibrinolysis in vivo is the diffusion of activator into thrombus with activation of intrinsic clot plasminogen in close association with fibrin, the substrative choice, which is digested (Sherry, Fletcher, and Alkjaersig, 1959a). In support of this hypothesis is the observation that streptokinase possesses the property of diffusing rapidly into thrombus (Gross, 1963). Sherry, Lindemeyer, Fletcher, and Alkjaersig (1959b) have demonstrated, using thrombi trace-labelled with radioactive fibrinogen, that thrombolysis is readily produced by activator on the surrounding medium but that thrombi are relatively resistant to plasmin in the surrounding medium. Further evidence of the importance of activation of intrinsic clot plasminogen is provided by the observation that rates of lysis of preformed radioactive clots are dependent on their plasminogen content.

We have recently compared the thrombolytic activity of urokinase, streptokinase, and plasmin and the coagulation defect produced by each of these agents (McNicol and Douglas, 1964). Constant volumes of saline, urokinase, streptokinase, or plasmin were added to fresh normal plasma. The fibrinolytic activity of the mixtures was assayed using the radioactive clot assay and the fibrin plate test. The thrombin clotting times and the proe thrombin times of the mixtures were also measureds With streptokinase and urokinase there was marked activity of thrombolytic activity in both tests, but, id the concentrations used, no effect on the thrombitio time or the prothrombin time as compared with the saline control. With the plasmin-plasma mixtureo however, no thrombolytic activity was found but there was a striking rise in the thrombin and proz thrombin times.

Activators as thrombolytic agents appear to reproduce the physiological mechanism of fibrino lysis and their ability to lyse preformed thrombus has been demonstrated in animal experiments, iro human experimental subjects (Johnson and McCart 1959), and in clinical trials (e.g., Verstreate an $\bar{\phi}$ Amery, 1962).

\section{UROKINASE}

This is a human protein and appears to be non? antigenic; compared with streptokinase it is als relatively non-toxic. Unfortunately, it is vers expensive, and although the price will no doubt falP with large-scale manufacture, it is unlikely ever to be cheap. Further and more important, the preparation available commercially at the present time (Leof urokinase) is not homogeneous, and both in vitrô and in vivo has been shown to have thromboplastie activity (McNicol, Gale, and Douglas, 1963). The effect can most readily be demonstrated using Hageman plasma as substrate. There is an inhibitof of urokinase in serum so that large doses have to be administered to produce a significant thrombolytiq effect in the plasma. For these reasons further work is required before urokinase can be submitted to widespread clinical trial.

\section{STREPTOKINASE}

Streptokinase, the first plasminogen activator to्? have become available, is still the most widely used but its main disadvantage is antigenicity. Antibodie to streptokinase are distributed throughout the population, varying very widely in concentration 
and until enough streptokinase has been given to neutralize circulating antibody, the specific biochemical effect on the fibrinolytic enzyme system is not seen. For this reason, when using streptokinase for systemic therapy it is essential to calculate an individual antibody-neutralizing dose for each patient. Streptokinase preparations at present are somewhat impure and tend to induce fever. There are formidable difficulties in the control of systemic therapy, although it is probable that more simple measures suffice for control of regional perfusion.

\section{NON-ENZYMATIC AGENTS}

Nicotinic acid (Weiner, de Crinis, Redisch, and Steele, 1959) and bacterial pyrogen (von Kaulla, 1963) both induce fibrinolytic activity in vivo, probably by bringing about release of activator into the circulation. Unfortunately with both agents resistance appears rapidly and there are troublesome side-effects.

Both chlorpropamide (Fearnley, Chakrabarti, and Vincent, 1960) and androgens (Fearnley and Chakrabarti, 1962) have been shown to cause an increase in fibrinolytic activity, and although the therapeutic implication of the observations has not as yet been adequately explored, these or similar substances may come to have an important role in prophylaxis.

\section{INDICATIONS FOR STREPTOKINASE THERAPY}

At the present time, despite its obvious disadvantages, streptokinase appears to be the most suitable agent for fibrinolytic therapy. There can be no question as to its potency as a thrombolytic agent in vitro and there is rapidly accumulating evidence as to its efficacy in vivo.

The main indication for streptokinase is probably recent peripheral arterial occlusion, and pulmonary embolism and retinal artery and vein thrombosis may also represent indications for streptokinase.

Contraindications are similar to those for anticoagulant therapy. Patients with peptic ulcer or other lesions liable to bleed should not be given streptokinase therapy and a recent cerebrovascular accident is also a contraindication. Because of the toxicity of the present preparations and the likely prolonged duration of infusion, streptokinase should not be given to seriously ill, old, or debilitated patients.

SELECTION OF CASES OF PERIPHERAL ARTERIAL OCCLUSION

Streptokinase treatment should be restricted to relatively fit patients with none of the contra- indications previously mentioned. It is also important that the occlusion should be recent, although the evidence on the relationship between the age of the thrombus and susceptibility to lysis is conflicting and inadequate, and this is an important field for further study. It would appear likely, as first suggested by Cotton, Flute, and Tsapogas (1962), that the most valuable role of streptokinase may be as a sequel to surgical removal of thrombus from small peripheral vessels beyond the reach of mechanical removal by the surgeon. Unless appropriate laboratory facilities and a suitable pump for intraarterial infusion at a constant rate are available, this form of therapy should not be contemplated.

LABORATORY CONTROL OF STREPTOKINASE THERAPY BY REGIONAL PERFUSION

In our view the essential measures for laboratory control of streptokinase therapy by regional perfusion at the present time include estimation of an initial antibody-neutralizing dose and then control of the subsequent infusion with the euglobulin lysis time and the thrombin clotting time. The infusion rate should be controlled at a level large enough to accelerate euglobulin lysis but not to prolong the thrombin time by more than 5 seconds. If possible, of course, fibrinogen and plasminogen assays should be carried out to extend understanding of the biochemical changes produced. A dosage rate which produces significant fibrinolytic activity in the circulation will almost certainly produce intense activity in the perfused limb. While further experience may show that it is not necessary to administer an initial antibody-neutralizing dose when streptokinase is being given by local perfusion, in the meantime to be certain of inducing a thrombolytic state an antibody-neutralizing dose should be given over one or two hours.

\section{PROBLEMS IN THROMBOLYTIC THERAPY}

We would like to conclude by reviewing briefly the current problems in thrombolytic therapy as we see them. The first and most important is, of course, the lack of suitable fibrinolytic agents and the cost and toxicity of those which are available. Laboratory control of therapy still presents a major problem, particularly for systemic therapy, though the evidence suggests that if streptokinase is given by local perfusion, simplified measures of control are adequate. Finally, there is the formidable problem of demonstrating clinical efficacy. Important here is case selection. Clinical investigation in this field must be restricted to problems where there is a prima facie case that benefit may be expected. It is 
obviously necessary to start treatment early and difficulties in this regard may be the all-important limiting factor, for example, in the treatment of coronary thrombosis. Just as important is the necessity to induce an adequate fibrinolytic state and to maintain this for an adequate period of time. The evidence suggests that in the treatment of peripheral occlusion fibrinolytic activity may need to be maintained for 30 or more hours, a formidable burden for the clinician and the laboratory.

However great these difficulties may appear, fibrinolytic therapy has many conceptual attractions and the problems in this field should be regarded as a challenge rather than as a deterrent.

We are grateful to the Medical Research Council for financial support. Streptokinase was kindly provided by Dr. Hans Dahlstrom, of Kabi Pharmaceutical, and urokinase by Dr. Austin Darragh, of Leo Laboratories.

\section{REFERENCES}

Cotton, L. T., Flute, P. T., and Tsapogas, M. J. C. (1962). Lancet, 2, 1081 .

Fearnley, G. R., and Chakrabarti, R. (1962). Lancet, 2, 128.

-, Chakrabarti, R., and Vincent, C. T. (1960). Ibid, 2, 622.

Gross, R. (1963). Proc. 9th Congr. of europ. Soc. Haematol., p. 142.
Johnson, A. J., and McCarty, W. R. (1959). J. clin. Invest., 38, 162 Kaulla, K. N. von. (1963). The Chemistry of Thrombolysis, p. 24 . Thomas, Springfield, Illinois.

McNicol, G. P., and Douglas, A. S. (1964). To be published.

Gale, S. B., and Douglas, A. S. (1963). Brit. med. J., 1, 909

Sawyer, W. D., Alkjaersig, N., Fletcher, A. P., and Sherry, S. (196/t Arch. intern. Med., 107, 274.

Sherry, S., and Fletcher, A. P. (1960). Clin. Pharmacol. Ther., 1, 2020 —_ _ , and Alkjaersig, N. (1959a). Physiol. Rev., 39, 343.

-, Lindemeyer, R. I., Fletcher, A. P., and Alkjaersig, N. (1959b局 J. clin. Invest., $38,810$.

Verstreate, M., and Amery, A. (1962). In Anticoagulant Therapy by A. S. Douglas, p. 357. Blackwell, Oxford.

Weiner, M., de Crinis, K., Redisch, W., and Steele, J. M. (1959) Circulation, 19, 845 .

DR. FLUTE felt that urokinase contained some clotting activity but that it was of minor degree. He and his colleagues had given urokinase to 17 dogs and two humans in quite high dosage and had not experience $\leftarrow$ any difficulty with thrombosis. He felt that for practicad purposes the clotting activity of urokinase was not disadvantage.

DR. MCNICOL believed that the difference between the views of Dr. Flute and himself was more apparent than real. When he said he thought that streptokinase appeared to be the best available preparation, this did not mean that he would necessarily hold this view in the future. Urokinase had been tried out to a much lessef extent than streptokinase and he had no doubt tha urokinase merited a much larger trial. 